\title{
Viability result for semilinear functional differential inclusions in Banach spaces
}

\begin{abstract}
Aitalioubrahim M.
We show the existence result of a mild solution for a semilinear functional differential inclusion, with viability, governed by a family of linear operators. We consider the case when the constraint is moving.

Key words and phrases: evolution operator, measurability, mild solution, functional differential inclusion, viability.
\end{abstract}

University Sultan Moulay Slimane, Bd Ibn Khaldoun, 23000 Beni-Mellal, Morocco

E-mail: aitalifrehotmail.com

\section{Introduction}

Let $E$ be a separable Banach space with the norm $\|\cdot\|$. For a segment $I$ in $\mathbb{R}$, we denote by $\mathcal{C}(I, E)$ the Banach space of continuous functions from $I$ to $E$ equipped with the norm $\|x(\cdot)\|_{\infty}:=\sup \{\|x(t)\|: t \in I\}$. For a positive number $a$, we put $\mathcal{C}_{a}:=\mathcal{C}([-a, 0], E)$ and for any $t \in[0, T], T>0$, we define the operator $T(t)$ from $\mathcal{C}([-a, T], E)$ to $\mathcal{C}_{a}$ with $(T(t)(x(\cdot)))(s):=(T(t) x)(s):=x(t+s), s \in[-a, 0]$.

In this paper, we shall prove the existence of solutions to the following semilinear functional differential inclusion

$$
\left\{\begin{array}{l}
\dot{x}(t) \in A(t) x(t)+F(t, T(t) x) \quad \text { a.e. on }[0, \tau], \\
x(s)=\varphi(s), \quad \forall s \in[-a, 0], \\
x(t) \in C(t), \quad \forall t \in[0, \tau],
\end{array}\right.
$$

where $F$ is a multifunction with closed values, measurable with respect to the first argument and Lipschitz continuous with respect to the second argument, $C$ is a multifunction with locally closed graph, $\{A(t): t \in[0, T]\}$ is a family of densely defined linear operators and $\varphi$ is a given function in $\mathcal{C}_{a}$.

O. Cârjă and I.I. Vrabie [5] have studied the problem $\dot{x}(t) \in A x(t)+F(x(t))$, where $F$ is a nonempty, closed, convex, and bounded valued mapping which is weakly-weakly upper semi-continuous. They have obtained a necessary and sufficient condition for the viability of a set K. In [4], O. Cârjă, M. Necula and I.I. Vrabie have proved another result for the same problem above.

Q. Dong and G. Li [8] have established the existence of solutions for (1) in the case, when $A(t)=A, F$ is a Carathéodory single valued-map and $C:] a, b\left[\rightarrow 2^{E}\right.$ is a set valued-map with closed values and such that, for each $\left.t_{0} \in\right] a, b\left[\right.$ and $x \in C\left(t_{0}\right)$, there exist $r>0$ and $\left.\tau \in\right] t_{0}, b[$ 
such that $B(x, r) \cap C(t)$ is nonempty for all $t \in\left[t_{0}, \tau\right]$, and the mapping $t \rightarrow B(x, r) \cap D(t)$ is closed on $\left[t_{0}, \tau\right]$.

V. Lupulescu and M. Necula, in [9], have established the existence of solutions for (1), when $A(t)=A, C(t)=C$ and $F$ is integrably bounded, measurable with respect to the first argument and Lipschitz continuous with respect to the second argument. The authors have used the following tangential condition

$$
\lim _{h \mapsto 0^{+}} \inf \frac{1}{h} e\left(S(h) \varphi(0)+\int_{t}^{t+h} S(t+h-s) F(s, \varphi) d s, C\right)=0, \quad \forall(t, \varphi) \in\left[a, b\left[\times \mathcal{K}_{0},\right.\right.
$$

where $e(\cdot, \cdot)$ denotes the Hausdorff's excess and $S(\cdot)$ is the $C_{0}$-semigroup generated by $A$.

It remains to notice that many viability results for (1), in the case $A=0$, have been proved in the papers $[1,2]$ and the references therein.

This work extends results, which are presented in $[1,2,8,9]$. Indeed, we get an existence result, in a separable Banach space, for semilinear functional differential inclusions with a constraint, which depends on time. The right-hand side verifies the weaker hypotheses. As is known, viability problems need tangential conditions. For the problem (1), we shall use a tangency condition, which is weaker than that used in [9].

The paper is organized as follows. In Section 1, we recall some preliminary facts that we need in the sequel. In Section 2, we prove the existence of solutions for (1).

\section{Notations and Preliminaries}

For measurability purpose, $E$ is endowed with the $\sigma$-algebra $B(E)$ of Borel subsets for the strong topology and $[0,1]$ is endowed with Lebesgue measure and the $\sigma$-algebra of Lebesgue measurable subsets. For $x \in E$ and $r>0$, let $B(x, r):=\{y \in E:\|y-x\|<r\}$ be the open ball centered at $x$ with radius $r$ and $\bar{B}(x, r)$ be its closure and put $B=B(0,1)$. For $\varphi(\cdot) \in \mathcal{C}_{a}$, let $B_{a}(\varphi(\cdot), r):=\left\{\psi(\cdot) \in \mathcal{C}_{a}:\|\varphi(\cdot)-\psi(\cdot)\|_{\infty}<r\right\}$ and let $\bar{B}_{a}(\varphi(\cdot), r)$ be its closure. For $x \in E$ and for nonempty subsets $A, B$ of $E$, we denote $d(x, A):=\inf \{\|y-x\|: y \in A\}$, $e(A, B):=\sup \left\{d_{B}(x): x \in A\right\}$ and $H(A, B):=\max \{e(A, B), e(B, A)\}$. We note $\mathcal{L}(E)$ the space of bounded linear operators on $E$. For any set-valued map $F$, we denote $G r(F)$ its graph. A multifunction is said to be measurable if its graph is measurable. For more details on measurability theory, we refer the reader to [7]. Now, let $\left\{A(t): D(A(t)) \rightarrow E, t \in \mathbb{R}^{+}\right\}$be a family of defined linear operators on $E$, where $D(A(t))$ is the domain of $A(t)$. We assume that for each $s \in \mathbb{R}^{+}$and $x \in E$, there is a unique solution $v:[s,+\infty[\rightarrow E$ for the evolution equation

$$
\begin{aligned}
& v^{\prime}(t)=A(t) v(t), \quad t \in[s,+\infty[, \\
& v(s)=x .
\end{aligned}
$$

In this case an operator $T(\cdot, \cdot)$ can be defined as

$$
T: \Delta=\left\{(t, s) \in \mathbb{R}^{2}: 0 \leq s \leq t\right\} \rightarrow \mathcal{L}(E), \quad T(t, s)(x)=v(t),
$$

where $v$ is the unique solution of (2). The operator $T(\cdot, \cdot)$ is called the evolution operator generated by the family $\left\{A(t): t \in \mathbb{R}^{+}\right\}$. It is clair that each operator $T(\cdot, \cdot)$ satisfies $T(s, s)=I_{E}$ and $T(t, r) T(r, s)=T(t, s)$ for all $0 \leq s \leq r \leq t$. Along this work, we consider an evolution operator $T(\cdot, \cdot)$, generated by a family $\left\{A(t): t \in \mathbb{R}^{+}\right\}$of defined linear operator on $E$, which satisfies 
(i) $\|T(t, s)\|_{\mathcal{L}(E)} \leq 1, \forall(t, s) \in \Delta$,

(ii) $(t, s) \mapsto T(t, s)$ is continuous.

Definition. By a mild solution of semilinear functional differential inclusion (1), we mean a continuous function $x(\cdot):[-a, \tau] \rightarrow$ E such that $x(\cdot)=\varphi$ on $[-a, 0]$ and

$$
x(t)=T(t, 0) \varphi(0)+\int_{0}^{t} T(t, s) f(s) d s, \quad \forall t \in[0, \tau],
$$

where $f$ is an integrable function such that $f(t) \in F(t, T(t) x)$, for almost every $t \in[0, \tau]$.

Let us recall the following Lemmas that will be used in the sequel.

Lemma 1 ([10]). Let $G:[a, b] \rightarrow 2^{E}$ be a measurable multifunction and $y(\cdot):[a, b] \rightarrow E$ a measurable function. Then for any positive measurable function $r(\cdot):[a, b] \rightarrow \mathbb{R}^{+}$, there exists a measurable selection $g(\cdot)$ of $G$ such that for almost all $t \in[a, b]$

$$
\|g(t)-y(t)\| \leq d(y(t), G(t))+r(t) .
$$

Lemma 2 ([3]). Let $\preceq$ be a given preorder on the nonempty set $\mathcal{B}$ and let $\phi: \mathcal{B} \rightarrow \mathbb{R} \cup\{+\infty\}$ be an increasing function. Suppose that each increasing sequence in $\mathcal{B}$ is majorated in $\mathcal{B}$. Then, for each $x_{0} \in \mathcal{B}$, there exists $x_{1} \in \mathcal{B}$ such that $x_{0} \preceq x_{1}$ and $\phi\left(x_{1}\right)=\phi(x)$ if $x_{1} \preceq x$.

The above function $\phi$, in [3], is supposed to be finite and bounded from above, but this restriction can be removed by replacing $\phi$ by the function $x \mapsto \arctan \phi(x)$ (see [6]).

In this paper, we shall use the following hypotheses.

(H1) $C:[0,1] \rightarrow 2^{E}$ is a set-valued map with locally closed graph and $\mathcal{K}:[0,1] \rightarrow \mathcal{C}_{a}$ is a set-valued map defined by $\mathcal{K}(t)=\left\{\varphi \in \mathcal{C}_{a}: \varphi(0) \in C(t)\right\}$.

$(\mathrm{H} 2) F: \operatorname{Gr}(\mathcal{K}) \rightarrow 2^{E}$ is a set-valued map with nonempty closed values satisfying:

(i) $t \mapsto F(t, \psi)$ is measurable,

(ii) there exists a function $m(\cdot) \in L^{1}\left([0,1], \mathbb{R}^{+}\right)$such that for all $t \in[0,1]$ and $\psi, \phi \in \mathcal{K}(t)$

$$
H(F(t, \psi), F(t, \phi)) \leq m(t)\|\psi-\phi\|_{\infty},
$$

(iii) there exist $g(\cdot), p(\cdot) \in L^{1}\left([0,1], \mathbb{R}^{+}\right)$such that for all $t \in[0,1]$ and $\psi \in \mathcal{K}(t)$

$$
\|F(t, \psi)\|:=\sup _{y \in F(t, \psi)}\|y\| \leq g(t)+p(t)\|\psi\|_{\infty} .
$$

(H3) (Tangential condition) For all measurable function $v(\cdot):[0,1] \rightarrow E$, for all $\rho>0, t \in[0,1]$ and $\psi \in \mathcal{K}(t)$, there exists $f \in S_{v, \rho}(\psi)$ such that

$$
\liminf _{h \mapsto 0^{+}} \frac{1}{h} d\left(T(t+h, t) \psi(0)+\int_{t}^{t+h} T(t+h, s) f(s) d s, C(t+h)\right)=0,
$$

where $S_{v, \rho}(\psi)$ is the set of all $f \in L^{1}([0,1], E)$ such that $f(s) \in F(s, \psi)$ for all $s \in[0,1]$ and $\|f(s)-v(s)\| \leq d(v(s), F(s, \psi))+\rho$ for almost all $s \in[0,1]$.

Remark. We should point out that, if $F$ satisfies the condition $(H 2)$, by Lemma 1 , the set $S_{v, \rho}(\psi)$ is nonempty.

We shall prove the following theorem.

Theorem. If assumptions (H1)-(H3) are satisfied, then for all $\varphi \in \mathcal{K}(0)$, there exist $\tau>0$ and a map $x(\cdot) \in \mathcal{C}([-a, \tau], E)$ such that $x(\cdot)$ is a mild solution of $(1)$. 


\section{Proof of the main result}

Let $\varphi \in \mathcal{K}(0)$ and set $x_{0}=\varphi(0)$. Since $\operatorname{Gr}(C)$ is locally closed, there exists $r>0$ such that $\operatorname{Gr}(C) \cap \bar{B}\left(\left(0, x_{0}\right), r\right)$ is closed. For $\varepsilon>0$ put

$$
\begin{aligned}
& \eta(\varepsilon):=\sup \{\rho \in] 0, \varepsilon]:\left\|\varphi\left(t_{1}\right)-\varphi\left(t_{2}\right)\right\|<\varepsilon,\left\|T\left(t_{1}, s_{1}\right)-T\left(t_{2}, s_{2}\right)\right\|_{\mathcal{L}(E)}<\varepsilon \\
& \text { and } \left.\left|\int_{t_{1}}^{t_{2}}\left(g(s)+p(s)\left(\|\varphi\|_{\infty}+r\right)\right) d s\right|<\varepsilon, \text { if }\left|t_{1}-t_{2}\right| \leq \rho \text { and }\left|s_{1}-s_{2}\right| \leq \rho\right\} .
\end{aligned}
$$

Let $\tau_{1}, \tau_{2}>0$ be such that

$$
\sup _{0 \leq t \leq \tau_{1}}\left\|T(t, 0) x_{0}-x_{0}\right\|+a \tau_{1}+\int_{0}^{\tau_{1}}\left(g(s)+p(s)\left(\|\varphi\|_{\infty}+r\right)\right) d s<\frac{r}{2} \text { and } \int_{0}^{\tau_{2}} m(t) d t<1 .
$$

Put $\tau=\inf \left\{\tau_{1}, \tau_{2},(1 / 2) \eta(r / 2), r / 2,1\right\}$. For all $0<\varepsilon<a$ and $v(\cdot) \in L^{1}([0,1], E)$, set $\mathcal{B}(\varepsilon, v(\cdot))$ the set of all 3-tuple $(f, x, \theta)_{d}$, where $\left.\left.d \in\right] 0, \tau\right], f(\cdot) \in L^{1}([0, d[, E), x(\cdot):[-a, d] \rightarrow E$ is a continuous function and $\theta(\cdot):[0, d[\rightarrow[0, d[$ is a step function such that:

(i) $f(t) \in F(t, T(\theta(t)) x), 0 \leq t-\theta(t) \leq(1 / 4) \eta(\varepsilon / 4), x(\theta(t)) \in C(\theta(t))$ and $T(\theta(t)) x \in$ $\bar{B}_{a}(\varphi, r)$ for all $t \in[0, d[$,

(ii) $x(d) \in C(d), T(d) x \in \bar{B}_{a}(\varphi, r), x(\cdot)=\varphi(\cdot)$ on $[-a, 0]$ and $x(t) \in \bar{B}\left(x_{0}, r / 2\right)$ for all $t \in[0, d]$

(iii) $\|f(t)-v(t)\| \leq d(v(t), F(t, T(\theta(t)) x))+\varepsilon$ for almost all $t \in[0, d[$,

(iv) for all $t \in[0, d]$

$$
\left\|x(t)-T(t, 0) x_{0}-\int_{0}^{t} T(t, s) f(s) d s\right\| \leq t \varepsilon
$$

Proposition. If assumptions (H1)-(H3) are satisfied, then for all $0<\varepsilon<a$, and $v(\cdot) \in$ $L^{1}([0,1], E)$, there exists at least one $(f, x, \theta)_{\tau} \in \mathcal{B}(\varepsilon, v(\cdot))$.

Proof. Let $0<\varepsilon<a$ and $v(\cdot) \in L^{1}([0,1], E)$ be fixed. Put $x(t)=\varphi(t) \forall t \in[-a, 0]$. By the tangential condition, there exist $f_{0} \in S_{v, \varepsilon}(\varphi)$ and $\left.\left.h_{0} \in\right] 0, \inf \{\tau,(1 / 4) \eta(\varepsilon / 4)\}\right]$, such that

$$
\frac{1}{h_{0}} d\left(T\left(h_{0}, 0\right) x_{0}+\int_{0}^{h_{0}} T\left(h_{0}, s\right) f_{0}(s) d s, C\left(h_{0}\right)\right) \leq \frac{\varepsilon}{2} .
$$

Then there exists $y_{0} \in C\left(h_{0}\right)$ such that

$$
\frac{1}{h_{0}}\left\|y_{0}-T\left(h_{0}, 0\right) x_{0}-\int_{0}^{h_{0}} T\left(h_{0}, s\right) f_{0}(s) d s\right\| \leq \varepsilon
$$

Set

$$
u_{0}=\frac{1}{h_{0}}\left(y_{0}-T\left(h_{0}, 0\right) x_{0}-\int_{0}^{h_{0}} T\left(h_{0}, s\right) f_{0}(s) d s\right) .
$$

We get

$$
y_{0}=T\left(h_{0}, 0\right) x_{0}+h_{0} u_{0}+\int_{0}^{h_{0}} T\left(h_{0}, s\right) f_{0}(s) d s
$$


Set $d_{0}=h_{0}$ and

$$
x_{0}(t)=T(t, 0) x_{0}+t u_{0}+\int_{0}^{t} T(t, s) f_{0}(s) d s \quad \forall t \in\left[0, d_{0}\right] .
$$

Remark that, for all $t \in\left[0, d_{0}\right]$

$$
\begin{aligned}
\left\|x_{0}(t)-x_{0}\right\| & \leq\left\|T(t, 0) x_{0}-x_{0}\right\|+h_{0} a+\int_{0}^{h_{0}}\left(g(s)+p(s)\|\varphi\|_{\infty}\right) d s \\
& \leq \sup _{0 \leq t \leq d_{0}}\left\|T(t, 0) x_{0}-x_{0}\right\|+d_{0} a+\int_{0}^{d_{0}}\left(g(s)+p(s)\left(\|\varphi\|_{\infty}+r\right)\right) d s \leq \frac{r}{2},
\end{aligned}
$$

then $x_{0}(t) \in \bar{B}\left(x_{0}, \frac{r}{2}\right)$ for all $t \in\left[0, d_{0}\right]$. Also, for all $t \in\left[0, d_{0}\right]$

$$
\left\|x_{0}(t)-T(t, 0) x_{0}-\int_{0}^{t} T(t, s) f_{0}(s) d s\right\| \leq t \varepsilon .
$$

Now, let $s \in[-a, 0]$. If $s \leq-d_{0}$, we have

$$
\left\|T\left(d_{0}\right) x(s)-\varphi(s)\right\|=\left\|x\left(s+d_{0}\right)-\varphi(s)\right\|=\left\|\varphi\left(s+d_{0}\right)-\varphi(s)\right\| \leq r,
$$

because $d_{0} \leq \frac{1}{2} \eta\left(\frac{r}{2}\right)$. If $s \geq-d_{0}$, one has

$$
\left\|T\left(d_{0}\right) x(s)-\varphi(s)\right\|=\left\|x\left(s+d_{0}\right)-\varphi(s)\right\| \leq\left\|x\left(s+d_{0}\right)-\varphi(0)\right\|+\|\varphi(s)-\varphi(0)\| \leq \frac{r}{2}+\frac{r}{2}=r,
$$

because $2|s| \leq \eta(r / 2)$ and $x\left(s+d_{0}\right) \in \bar{B}\left(x_{0}, r / 2\right)$. From the above, we conclude that $T\left(d_{0}\right) x \in$ $\bar{B}_{a}(\varphi, r)$. Next, set $\theta_{0}(t)=0$ for all $t \in\left[0, d_{0}\right.$ [. It is clair that $\left(f_{0}, x_{0}, \theta_{0}\right)_{d_{0}} \in \mathcal{B}(\varepsilon, v(\cdot))$. Hence $\mathcal{B}(\varepsilon, v(\cdot)) \neq \varnothing$. Now, consider the following preorder:

$$
\left(f_{1}, x_{1}, \theta_{1}\right)_{d_{1}} \preceq\left(f_{2}, x_{2}, \theta_{2}\right)_{d_{2}} \Leftrightarrow d_{1} \leq d_{2}, \quad f_{1}=\left.f_{2}\right|_{\left[0, d_{1}[\right.}, \quad x_{1}=\left.x_{2}\right|_{\left[0, d_{1}\right]},
$$

and $\theta_{1}=\left.\theta_{2}\right|_{\left[0, d_{1}[\right.}$. Let $\phi: \mathcal{B}(\varepsilon, v(\cdot)) \rightarrow \mathbb{R}$ be the function defined by $\phi\left((f, x, \theta)_{d}\right)=d$, $\forall(f, x, \theta)_{d} \in \mathcal{B}(\varepsilon, v(\cdot))$. By definition, $\phi$ is increasing on $\mathcal{B}(\varepsilon, v(\cdot))$. On the other hand, if $\left(\left(f_{i}, x_{i}, \theta_{i}\right)_{d_{i}}\right)_{i \in \mathbb{N}}$ is an increasing sequence in $\mathcal{B}(\varepsilon, v(\cdot))$, we construct a majorant $(f, x, \theta)_{d}$ of $\left(\left(f_{i}, x_{i}, \theta_{i}\right)_{d_{i}}\right)_{i \in \mathbb{N}}$ as follows: $d=\lim _{i} d_{i}, f(t)=f_{i}(t), \theta(t)=\theta_{i}(t)$ for all $t \in\left[0, d_{i}[, x(\cdot)=\varphi(\cdot)\right.$ on $[-a, 0], x(t)=x_{i}(t)$ for all $t \in\left[0, d_{i}\right]$. We claim that $(f, x, \theta)_{d} \in \mathcal{B}(\varepsilon, v(\cdot))$. Indeed, for all $i \in \mathbb{N}$, we have $x\left(d_{i}\right)=x_{i}\left(d_{i}\right) \in C\left(d_{i}\right)$. Then $\left(d_{i}, x\left(d_{i}\right)\right) \in G r(C) \cap \bar{B}\left(\left(0, x_{0}\right), r\right)$, for all $i \in \mathbb{N}$. Since $\operatorname{Gr}(C) \cap \bar{B}\left(\left(0, x_{0}\right), r\right)$ is closed, we conclude that $(d, x(d)) \in G r(C) \cap \bar{B}\left(\left(0, x_{0}\right), r\right)$. By the same argument, we get $x(d) \in \bar{B}\left(x_{0}, r / 2\right)$. For (iv), it is clair that

$$
\left\|x(t)-T(t, 0) x_{0}-\int_{0}^{t} T(t, s) f(s) d s\right\| \leq t \varepsilon, \quad \forall t \in[0, d[.
$$

In addition, by the fact that the function $T(\cdot, \cdot)$ is continuous, one has

$$
\lim _{i \rightarrow+\infty}\left\|x\left(d_{i}\right)-T\left(d_{i}, 0\right) x_{0}-\int_{0}^{d_{i}} T\left(d_{i}, s\right) f(s) d s\right\|=\left\|x(d)-T(d, 0) x_{0}-\int_{0}^{d} T(d, s) f(s) d s\right\| .
$$

Hence, we get

$$
\left\|x(d)-T(d, 0) x_{0}-\int_{0}^{d} T(d, s) f(s) d s\right\| \leq d \varepsilon .
$$

The other assertions are obvious. Next, for applying Lemma 2, we need the following claim. 
Claim 1. For all $(f, x, \theta)_{d} \in \mathcal{B}(\varepsilon, v(\cdot))$ with $d<\tau$, there exists $(\bar{f}, \bar{x}, \bar{\theta})_{\bar{d}} \in \mathcal{B}(\varepsilon, v(\cdot))$ such that $(f, x, \theta)_{d} \preceq(\bar{f}, \bar{x}, \bar{\theta})_{\bar{d}}$ and $\phi\left((f, x, \theta)_{d}\right)<\phi\left((\bar{f}, \bar{x}, \bar{\theta})_{\bar{d}}\right)$.

Proof. Let $(f, x, \theta)_{d} \in \mathcal{B}(\varepsilon, v(\cdot))$ with $d<\tau$. For $T(d) x \in \mathcal{K}(d)$, by the tangential condition, there exist $\tilde{f} \in S_{v, \varepsilon}(T(d) x)$ and $\left.\left.h \in\right] 0, \inf \{\tau-d,(1 / 4) \eta(\varepsilon / 4)\}\right]$, such that

$$
\frac{1}{h} d\left(T(d+h, d) x(d)+\int_{d}^{d+h} T(d+h, s) \tilde{f}(s) d s, C(d+h)\right) \leq \frac{\varepsilon}{2} .
$$

Then there exists $y_{1} \in C(d+h)$ such that

$$
\frac{1}{h}\left\|y_{1}-T(d+h, d) x(d)-\int_{d}^{d+h} T(d+h, s) \tilde{f}(s) d s\right\| \leq \varepsilon
$$

Set

$$
u_{1}=\frac{1}{h}\left(y_{1}-T(d+h, d) x(d)-\int_{d}^{d+h} T(d+h, s) \tilde{f}(s) d s\right) .
$$

We have

$$
y_{1}=T(d+h, d) x(d)+h u_{1}+\int_{d}^{d+h} T(d+h, s) \tilde{f}(s) d s .
$$

Next, set $\bar{d}=d+h$,

$$
\tilde{x}(t)=T(t, d) x(d)+(t-d) u_{1}+\int_{d}^{t} T(t, s) \tilde{f}(s) d s, \quad \forall t \in[d, \bar{d}] .
$$

We define $\bar{f}, \bar{\theta}$ and $\bar{x}$ as follows: $\bar{f}(t)=f(t), \bar{\theta}(t)=\theta(t)$ for all $t \in[0, d[, \bar{f}(t)=\tilde{f}(t), \bar{\theta}(t)=d$ for all $t \in[d, \bar{d}[, \bar{x}(\cdot)=\varphi(\cdot)$ on $[-a, 0], \bar{x}(t)=x(t)$ for all $t \in[0, d]$ and $\bar{x}(t)=\tilde{x}(t)$ for all $t \in[d, \bar{d}]$. Now, for all $t \in[d, \bar{d}]$, we have

$$
\begin{aligned}
\| \bar{x}(t)-T(t, 0) x_{0} & -\int_{0}^{t} T(t, s) \bar{f}(s) d s \| \\
& =\left\|T(t, d) x(d)+(t-d) u_{1}+\int_{d}^{t} T(t, s) \tilde{f}(s) d s-T(t, 0) x_{0}-\int_{0}^{t} T(t, s) \bar{f}(s) d s\right\| \\
& =\left\|T(t, d)\left(x(d)-T(d, 0) x_{0}-\int_{0}^{d} T(d, s) f(s) d s\right)+(t-d) u_{1}\right\| \\
& \leq\|T(t, d)\|_{\mathcal{L}(E)} d \varepsilon+(t-d) \varepsilon \leq t \varepsilon
\end{aligned}
$$

and by (3)

$$
\begin{aligned}
\left\|\bar{x}(t)-x_{0}\right\| & \leq\left\|\bar{x}(t)-T(t, 0) x_{0}-\int_{0}^{t} T(t, s) \bar{f}(s) d s\right\|+\left\|T(t, 0) x_{0}-x_{0}+\int_{0}^{t} T(t, s) \bar{f}(s) d s\right\| \\
& \leq t \varepsilon+\sup _{0 \leq s \leq \tau}\left\|T(s, 0) x_{0}-x_{0}\right\|+\int_{0}^{\bar{d}}(g(s)+p(s)\|T(\bar{\theta}(s)) \bar{x}\|) d s \\
& \leq \sup _{0 \leq s \leq \tau}\left\|T(s, 0) x_{0}-x_{0}\right\|+a \tau+\int_{0}^{\tau}\left(g(s)+p(s)\left(\|\varphi\|_{\infty}+r\right)\right) d s \leq \frac{r}{2},
\end{aligned}
$$

then $\bar{x}(t) \in \bar{B}\left(x_{0}, r / 2\right)$ for all $t \in[0, \bar{d}]$. Now, let $s \in[-a, 0]$. If $s \leq-\bar{d}$, we have

$$
\|T(\bar{d}) \bar{x}(s)-\varphi(s)\|=\|\bar{x}(s+\bar{d})-\varphi(s)\|=\|\varphi(s+\bar{d})-\varphi(s)\| \leq r .
$$


If $s \geq-\bar{d}$, one has

$$
\|T(\bar{d}) x(s)-\varphi(s)\|=\|\bar{x}(s+\bar{d})-\varphi(s)\| \leq\|\bar{x}(s+\bar{d})-\varphi(0)\|+\|\varphi(s)-\varphi(0)\| \leq r .
$$

Hence $T(\bar{d}) \bar{x} \in \bar{B}_{a}(\varphi, r)$. Finally, we conclude that $(\bar{f}, \bar{x}, \bar{\theta})_{\bar{d}} \in \mathcal{B}(\varepsilon, v(\cdot)),(f, x, \theta)_{d} \preceq(\bar{f}, \bar{x}, \bar{\theta})_{\bar{d}}$ and $\phi\left((f, x, \theta)_{d}\right)<\phi\left((\bar{f}, \bar{x}, \bar{\theta})_{\bar{d}}\right)$.

Now, we are ready to complete the proof of proposition. From Lemma 2, there exists $(f, x, \theta)_{d} \in \mathcal{B}(\varepsilon, v(\cdot))$ such that $\phi\left((f, x, \theta)_{d}\right)=\phi\left((\bar{f}, \bar{x}, \bar{\theta})_{\bar{d}}\right)$ and $(f, x, \theta)_{d} \preceq(\bar{f}, \bar{x}, \bar{\theta})_{\bar{d}}$ for all $(\bar{f}, \bar{x}, \bar{\theta})_{\bar{d}} \in \mathcal{B}(\varepsilon, v(\cdot))$. Moreover, if $\phi\left((f, x, \theta)_{d}\right)<\tau$, by the Claim 1, there exists $(\bar{f}, \bar{x}, \bar{\theta})_{\bar{d}} \in \mathcal{B}(\varepsilon, v(\cdot))$ such that $(f, x, \theta)_{d} \preceq(\bar{f}, \bar{x}, \bar{\theta})_{\bar{d}}$ and $\phi\left((f, x, \theta)_{d}\right)<\phi\left((\bar{f}, \bar{x}, \bar{\theta})_{\bar{d}}\right)$. Hence, $\phi\left((f, x, \theta)_{d}\right)=\tau$.

In the next, we will prove our theorem. Let $\left(\varepsilon_{n}\right)_{n \geq 1}$ be a strictly decreasing sequence of positive scalars such that $0<\varepsilon_{n}<a$ for all $n \geq 1$ and $\sum_{n=1}^{\infty} \varepsilon_{n}<\infty$. In view of Proposition, we can define inductively sequences $\left(f_{n}(\cdot)\right)_{n \geq 1} \subset L^{1}\left(\left[0, \tau[, E),\left(x_{n}(\cdot)\right)_{n \geq 1} \subset \mathcal{C}([-a, \tau], E)\right.\right.$, and $\left(\theta_{n}(\cdot)\right)_{n \geq 1} \subset S([0, \tau[,[0, \tau[)$, where $S([0, \tau[,[0, \tau[)$ denotes the space of step functions from $[0, \tau[$ into $[0, \tau[$ such that:

(a) $f_{n}(t) \in F\left(t, T\left(\theta_{n}(t)\right) x_{n}\right), x_{n}\left(\theta_{n}(t)\right) \in C\left(\theta_{n}(t)\right), T\left(\theta_{n}(t)\right) x_{n} \in \bar{B}_{a}(\varphi, r)$ and $0 \leq t-\theta_{n}(t) \leq$ $(1 / 4) \eta\left(\varepsilon_{n} / 4\right)$ for all $t \in[0, \tau[$,

(b) $x_{n}(\tau) \in C(\tau), T(\tau) x_{n} \in \bar{B}_{a}(\varphi, r)$ and $x_{n}(t) \in \bar{B}\left(x_{0}, r / 2\right)$ for all $t \in[0, \tau]$ and $x_{n} \equiv \varphi$ on $[-a, 0]$

(c) $\left\|f_{n+1}(t)-f_{n}(t)\right\| \leq d\left(f_{n}(t), F\left(t, T\left(\theta_{n+1}(t)\right) x_{n+1}\right)\right)+\varepsilon_{n+1}$ for almost all $t \in[0, \tau[$,

(d) for all $t \in[0, \tau]$

$$
\left\|x_{n}(t)-T(t, 0) x_{0}-\int_{0}^{t} T(t, s) f_{n}(s) d s\right\| \leq \varepsilon_{n} t
$$

In the rest of this paper, we take $\theta_{n}(\tau)=\tau$ for all $n \geq 1$ and we denote the modulus continuity of a function $\psi$ defined on interval $I$ of $\mathbb{R}$ by

$$
\omega(\psi, I, \varepsilon):=\sup \{\|\psi(t)-\psi(s)\|: s, t \in I,|s-t|<\varepsilon\}, \quad \varepsilon>0
$$

Claim 2. For all $t \in[0, \tau]$

$$
\left\|T\left(\theta_{n}(t)\right) x_{n}-T\left(\theta_{n+1}(t)\right) x_{n+1}\right\|_{\infty} \leq \varepsilon_{n} \delta+\left\|x_{n+1}(\cdot)-x_{n}(\cdot)\right\|_{\infty},
$$

where

$$
\delta=4+\left\|x_{0}\right\|+\int_{0}^{\tau}\left(g(s)+p(s)\left(\|\varphi\|_{\infty}+r\right)\right) d s .
$$


Proof. First, for $t, t^{\prime} \in[0, \tau]$ with $0 \leq t^{\prime}-t \leq(1 / 2) \eta\left(\varepsilon_{n} / 4\right)$, one has

$$
\begin{aligned}
& \left\|x_{n+1}(t)-x_{n+1}\left(t^{\prime}\right)\right\| \leq\left\|x_{n+1}(t)-T(t, 0) x_{0}-\int_{0}^{t} T(t, s) f_{n+1}(s) d s\right\| \\
& +\left\|T(t, 0) x_{0}+\int_{0}^{t} T(t, s) f_{n+1}(s) d s-T\left(t^{\prime}, 0\right) x_{0}-\int_{0}^{t^{\prime}} T\left(t^{\prime}, s\right) f_{n+1}(s) d s\right\| \\
& +\left\|x_{n+1}\left(t^{\prime}\right)-T\left(t^{\prime}, 0\right) x_{0}-\int_{0}^{t^{\prime}} T\left(t^{\prime}, s\right) f_{n+1}(s) d s\right\| \\
& \leq 2 \varepsilon_{n+1}+\left\|T(t, 0)-T\left(t^{\prime}, 0\right)\right\|_{\mathcal{L}(E)}\left\|x_{0}\right\| \\
& +\int_{0}^{t}\left\|T(t, s)-T\left(t^{\prime}, s\right)\right\|_{\mathcal{L}(E)}\left\|f_{n+1}(s)\right\| d s+\int_{t}^{t^{\prime}}\left\|T\left(t^{\prime}, s\right)\right\|_{\mathcal{L}(E)}\left\|f_{n+1}(s)\right\| d s \\
& \leq 2 \varepsilon_{n+1}+\varepsilon_{n}\left\|x_{0}\right\|+\varepsilon_{n} \int_{0}^{\tau}\left(g(s)+p(s)\left(\|\varphi\|_{\infty}+r\right)\right) d s \\
& +\int_{t}^{t^{\prime}}\left(g(s)+p(s)\left(\|\varphi\|_{\infty}+r\right)\right) d s \\
& \leq \varepsilon_{n}\left(3+\left\|x_{0}\right\|+\int_{0}^{\tau}\left(g(s)+p(s)\left(\|\varphi\|_{\infty}+r\right)\right) d s\right) .
\end{aligned}
$$

Hence,

$$
\omega\left(x_{n+1}(\cdot),[0, \tau], \frac{1}{2} \eta\left(\frac{\varepsilon_{n}}{4}\right)\right) \leq \varepsilon_{n}\left(3+\left\|x_{0}\right\|+\int_{0}^{\tau}\left(g(s)+p(s)\left(\|\varphi\|_{\infty}+r\right)\right) d s\right) .
$$

Now, for all $t \in[0, \tau]$, we have

$$
\begin{aligned}
\left\|T\left(\theta_{n+1}(t)\right) x_{n+1}-T\left(\theta_{n}(t)\right) x_{n}\right\|_{\infty}= & \sup _{s \in[-a, 0]}\left\|x_{n+1}\left(\theta_{n+1}(t)+s\right)-x_{n}\left(\theta_{n}(t)+s\right)\right\| \\
\leq & \sup _{s \in[-a, 0]}\left\|x_{n+1}\left(\theta_{n+1}(t)+s\right)-x_{n+1}\left(\theta_{n}(t)+s\right)\right\| \\
& +\sup _{s \in[-a, 0]}\left\|x_{n+1}\left(\theta_{n}(t)+s\right)-x_{n}\left(\theta_{n}(t)+s\right)\right\| \\
\leq & \omega\left(x_{n+1}(\cdot),[-a, \tau], \frac{1}{2} \eta\left(\frac{\varepsilon_{n}}{4}\right)\right)+\sup _{s \in[-a, \tau]}\left\|x_{n+1}(s)-x_{n}(s)\right\| \\
\leq & \omega\left(x_{n+1}(\cdot),[-a, 0], \frac{1}{2} \eta\left(\frac{\varepsilon_{n}}{4}\right)\right)+\omega\left(x_{n+1}(\cdot),[0, \tau], \frac{1}{2} \eta\left(\frac{\varepsilon_{n}}{4}\right)\right) \\
& +\left\|x_{n+1}(\cdot)-x_{n}(\cdot)\right\|_{\infty} \\
\leq & \frac{\varepsilon_{n}}{4}+\varepsilon_{n}\left(3+\left\|x_{0}\right\|+\int_{0}^{\tau}\left(g(s)+p(s)\left(\|\varphi\|_{\infty}+r\right)\right) d s\right) \\
& +\left\|x_{n+1}(\cdot)-x_{n}(\cdot)\right\|_{\infty} \\
\leq & \varepsilon_{n} \delta+\left\|x_{n+1}(\cdot)-x_{n}(\cdot)\right\|_{\infty} .
\end{aligned}
$$

Now, from (a) and (c), we deduce for almost all $t \in[0, \tau[$

$$
\begin{aligned}
\left\|f_{n+1}(t)-f_{n}(t)\right\| & \leq H\left(F\left(t, T\left(\theta_{n}(t)\right) x_{n}\right), F\left(t, T\left(\theta_{n+1}(t)\right) x_{n+1}\right)\right)+\varepsilon_{n+1} \\
& \left.\leq m(t) \| T\left(\theta_{n}(t)\right) x_{n}-T\left(\theta_{n+1}(t)\right) x_{n+1}\right) \|_{\infty}+\varepsilon_{n+1} \\
& \leq m(t) \varepsilon_{n} \delta+m(t)\left\|x_{n+1}(\cdot)-x_{n}(\cdot)\right\|_{\infty}+\varepsilon_{n+1} .
\end{aligned}
$$


On the other hand, for all $t \in[0, \tau]$, we have

$$
\begin{aligned}
\left\|x_{n+1}(t)-x_{n}(t)\right\| \leq & \left\|x_{n+1}(t)-T(t, 0) x_{0}-\int_{0}^{t} T(t, s) f_{n+1}(s) d s\right\| \\
& +\left\|T(t, 0) x_{0}+\int_{0}^{t} T(t, s) f_{n+1}(s) d s-T(t, 0) x_{0}-\int_{0}^{t} T(t, s) f_{n}(s) d s\right\| \\
& +\left\|x_{n}(t)-T(t, 0) x_{0}-\int_{0}^{t} T(t, s) f_{n}(s) d s\right\| \\
\leq & 2 \varepsilon_{n}+\int_{0}^{t}\left\|f_{n+1}(s)-f_{n}(s)\right\| d s \\
\leq & 3 \varepsilon_{n}+\varepsilon_{n} \delta \int_{0}^{\tau} m(s) d s+\left\|x_{n+1}(\cdot)-x_{n}(\cdot)\right\|_{\infty} \int_{0}^{\tau} m(s) d s .
\end{aligned}
$$

Thus

$$
\left\|x_{n+1}(\cdot)-x_{n}(\cdot)\right\|_{\infty} \leq \frac{\varepsilon_{n}(3+\delta)}{1-L}, \quad L=\int_{0}^{\tau} m(s) d s .
$$

Therefore we have, for $n<m$,

$$
\left\|x_{n}(\cdot)-x_{m}(\cdot)\right\|_{\infty} \leq \frac{3+\delta}{1-L} \sum_{j=n}^{m-1} \varepsilon_{j} .
$$

So the sequence $\left\{x_{n}(\cdot)\right\}_{n=1}^{\infty}$ is a Cauchy sequence, then it converges uniformly on $[0, \tau]$ to a function $x(\cdot)$. Since all functions $x_{n}(\cdot)$ agree with $\varphi$ on $[-a, 0]$, we can obviously say that $x_{n}(\cdot)$ converges uniformly to $x(\cdot)$ on $[-a, \tau]$, if we extend $x(\cdot)$ in such a way that $x(\cdot) \equiv \varphi$ on $[-a, 0]$. Also, by the following inequality

$$
\left\|x_{n}\left(\theta_{n}(t)\right)-x(t)\right\| \leq \omega\left(x_{n}(\cdot),[0, \tau], \frac{1}{2} \eta\left(\frac{\varepsilon_{n}}{4}\right)\right)+\left\|x_{n}(t)-x(t)\right\|,
$$

we deduce that $x_{n}\left(\theta_{n}(\cdot)\right)$ converges uniformly to $x(\cdot)$ on $[0, \tau]$. By construction, we have $\left(\theta_{n}(t), x_{n}\left(\theta_{n}(t)\right)\right) \in G r(C) \cap \bar{B}\left(\left(0, x_{0}\right), r\right)$ for every $t \in[0, \tau]$, then $x(t) \in C(t)$ for all $t \in[0, \tau]$. Now, we return to the relation (5). By the relation (6), for almost all $t \in[0, \tau$, we get

$$
\left\|f_{n+1}(t)-f_{n}(t)\right\| \leq \varepsilon_{n}\left(m(t)\left(\delta+\frac{3+\delta}{1-L}\right)+1\right)
$$

This implies (as above) that $\left\{f_{n}(t)\right\}_{n=1}^{\infty}$ is a Cauchy sequence and $\left(f_{n}(t)\right)_{n}$ converges to $f(t)$ for almost all $t \in[0, \tau[$. Further, since

$$
\left.\left\|f_{n}(t)\right\| \leq g(t)+p(t)\left(\|\varphi\|_{\infty}+r\right)\right), \quad \forall t \in[0, \tau[,
$$

by (d) and by the dominated convergence theorem, for all $t \in[0, \tau]$, we get

$$
x(t)=\lim _{n \rightarrow \infty} x_{n}(t)=\lim _{n \rightarrow \infty}\left(T(t, 0) x_{0}+\int_{0}^{t} T(t, s) f_{n}(s) d s\right)=T(t, 0) x_{0}+\int_{0}^{t} T(t, s) f(s) d s .
$$

In the rest, we will show that $f(t) \in F(t, T(t) x)$ a.e on $[0, \tau]$. First, let $t \in[0, \tau]$. By (4), we have

$$
\begin{aligned}
\left\|T\left(\theta_{n}(t)\right) x_{n}-T(t) x_{n}\right\|_{\infty} & =\sup _{-a \leq s \leq 0}\left\|x_{n}\left(\theta_{n}(t)+s\right)-x_{n}(t+s)\right\| \leq \omega\left(x_{n},[-a, \tau], \frac{1}{2} \eta\left(\frac{\varepsilon_{n}}{4}\right)\right) \\
& \leq \omega\left(\varphi,[-a, 0], \frac{1}{2} \eta\left(\frac{\varepsilon_{n}}{4}\right)\right)+\omega\left(x_{n},[0, \tau], \frac{1}{2} \eta\left(\frac{\varepsilon_{n}}{4}\right)\right) \\
& \leq \frac{\varepsilon_{n}}{4}+\varepsilon_{n}\left(3+\left\|x_{0}\right\|+\int_{0}^{\tau}\left(g(s)+p(s)\left(\|\varphi\|_{\infty}+r\right)\right) d s\right) \leq \varepsilon_{n} \delta .
\end{aligned}
$$


Hence $\left\|T\left(\theta_{n}(t)\right) x_{n}-T(t) x_{n}\right\|_{\infty}$ converges to 0 as $n \rightarrow+\infty$. Therefore, since the uniform convergence of $x_{n}(\cdot)$ to $x(\cdot)$ on $[-a, \tau]$ implies that $T(t) x_{n}$ converges to $T(t) x$ uniformly on $[-a, 0]$, we deduce that $T\left(\theta_{n}(t)\right) x_{n}$ converges to $T(t) x$ in $\mathcal{C}_{a}$. Now, observe that by (a),

$$
\begin{aligned}
d(f(t), F(t, T(t) x)) & \leq H\left(F\left(t, T\left(\theta_{n}(t)\right) x_{n}\right), F(t, T(t) x)\right)+\left\|f(t)-f_{n}(t)\right\| \\
& \leq m(t)\left\|T\left(\theta_{n}(t)\right) x_{n}-T(t) x\right\|_{\infty}+\left\|f(t)-f_{n}(t)\right\|
\end{aligned}
$$

for almost all $t \in[0, \tau[$. Since the last term converges to 0 , we get $f(t) \in F(t, T(t) x)$ a.e on $[0, \tau]$.

\section{Acknowledgements}

The author would like to thank the referee for his careful and thorough reading of the paper.

\section{References}

[1] Aitalioubrahim M. A viabillity result for functional differential inclusions in Banach spaces. Miskolc Math. Notes 2012, 13 (1), 3-22. doi:10.18514/MMN.2012.334

[2] Aitalioubrahim M. On viability result for first order functional differential inclusions. Mat. Vesnik 2018 , 70 (4), 283-291.

[3] Brézis H., Brouder F.E. A general principle on ordered sets in nolinear functional analysis. Adv. Math. 1976, 21 (3), 355-364. doi:10.1016/S0001-8708(76)80004-7

[4] Cârjă O., Necula M., Vrabie I.I. Necessary and sufficient conditions for viability for semilinear differential inclusions. Trans. Amer. Math. Soc. 2009, 361 (1), 343-390.

[5] Cârjă O., Vrabie I.I. Viability for semilinear differential inclusions via the weak sequential tangency condition. J. Math. Anal. Appl. 2001, 262 (1), 24-38. doi:10.1006/jmaa.2001.7477

[6] Cârjă O., Ursescu C. The characteristics method for a first order partial differential equation. An. Stiint. Univ. Al. I. Cuza Iasi. Mat. 1993, 39, 367-396.

[7] Castaing C., Valadier M. Convex analysis and measurable multifunctions. Lecture Notes in Mathematics, 580. Springer, Berlin, Heidelberg, 1977.

[8] Dong Q., Li G. Viability for semilinear differential equations with infinite delay. Math. 2016, 4 (4), 64. doi: 10.3390/math4040064

[9] Lupulescu V., Necula M. A viability result for nonconvex semilinear functional differential inclusions. Discuss. Math. Differ. Incl. Control Optim. 2005, 25, 109-128.

[10] Zhu Q. On the solution set of differential inclusions in Banach spaces. J. Differential Equations 1991, 93 (2), 213 237. doi:10.1016/0022-0396(91)90011-W

Received 20.10.2020

Revised 05.02.2021

Айталіубрахім М. Результат життездатності напівлінійних функціонально-диференціальних включень у банахових просторах // Карпатські матем. публ. - 2021. - Т.13, №2. - С. 395-404.

У роботі показано результат існування м'якого розв'язку напівлінійного функціональнодиференціального включення з життєздатністю, що регулюється сім'єю лінійних операторів, у випадку рухомого обмеження.

Ключові слова і фрази: оператор еволюції, вимірюваність, м'який розв'язок, функціональнодиференціальне включення, життєздатність. 